\title{
A grouping interpretation of the modality effect in immediate probed recognition
}

\author{
DAVID J. MURRAY, NICOLE BOUDREAU, KIMBERLEY K. BURGGRAF, \\ LESLIE DOBELL, SHARON L. GUGER, ANDREA LEASK, \\ LIANNE STANFORD, TRICIA L. TATE, and MELISSA WHEELER \\ Queen's University, Kingston, Ontario, Canada
}

\begin{abstract}
In a series of experiments on immediate probed recognition for eight 3-digit numbers, it was shown that if the target modality involved auditory components and the effect of the similarity of the modality of the probe to that of the targets was controlled, unequivocal evidence was obtained for an auditory superiority effect (modality effect) for hit rates for the final items of the list. Moreover, false-alarm rates were significantly lower following targets with an auditory component than they were following silently seen targets. It is argued that this pattern of hits and false alarms is consistent with the idea that targets that have an auditory component yield memory representations that are better grouped as units than are those for targets that are only silently seen; in particular, if a new probe has a first digit that accidentally matches the first digit of a target item, it is more likely that the subject will mistakenly identify this new probe as old (give a false alarm) if the target has only been partially encoded because it was only silently seen.
\end{abstract}

A typical experimental procedure in the immediate probed recognition paradigm, first studied in depth by Wickelgren and Norman (1966), involves presenting a sequence of three-digit numbers, such as 318 or 296 (target digit-triples), followed by a probe digit-triple that is either a repetition of one of the target triples (i.e., is old) or is not (i.e., is new). The subject must judge the probe to be either old or new, and Wickelgren and Norman showed how the probability of a correct old judgment (a hit) decreased as list length increased, because, as each

This research consists mainly of honors theses that were supervised by D.J.M. A preliminary version of partial matching theory, including the data here reported, was first presented at a colloquium in the Department of Psychology at the University of New Hampshire on April 24, 1997; the first author is indebted to the audience for its helpful feedback. D. E. Birch assisted substantially in the development of the computer program, originally designed by Lorraine Farrell, that controlled the presentation of the targets and probes and summarized the subjects' responses in terms of hit rates, false-alarm rates, and other summary statistics. Sylvia Hains carried out all the analyses of variance and calculations of $d_{1}, P_{r}, C_{\mathrm{L}}, B_{r}$, and standard errors, using the Queen's University mainframe computer, and Ron Holden gave us exact estimates of probabilities associated with correlation coefficients calculated in the course of this and other studies of probed recognition. The personal computers used in these experiments were also provided by Queen's University. Maureen Freedman and Rachel Murray typed the manuscript, Bahar Farahmand aided substantially in drafting the manuscript and in reanalyzing data collected earlier, and Monica Hurt did the illustrations. We also wish to acknowledge the valuable help given by John Gardiner and an anonymous reviewer with respect to the first submission of the present paper, by John Wixted with respect to the final version, and by Robert Greene, Ian Neath, Bennet B. Murdock, and Peter Dixon, all of whom were constructive reviewers of earlier descriptions of this research. Correspondence concerning this article should be sent to D. J. Murray, Department of Psychology, Queen's University, Kingston, ON K7L 3N6, Canada (e-mail: murrayd@pavlov. psyc.queensu.ca). new target item appeared, it appeared to cause some forgetting of previous target items. In their experiments, the probability of an old probe was .67 , and presentation was auditory (by a tape recording), with the triples being heard at a rate of one digit-triple per second. They also used $d^{\prime}$ as a more refined measure of discrimination, taking account of the false-alarm (FA) rate, as given by the probability of an incorrect old response to a new probe. Subsequent experiments by Murray and his colleagues, in which the probability of an old probe was set at .5, demonstrated that performance, as measured by $d^{\prime}$ values on probed recognition with auditory digit-duples presented at that rate, was lower in patients with Korsakoff's psychosis than it was in alcoholic control subjects (Murray \& Hitchcock, 1974); with auditory digit-triples, $d^{\prime}$ was lower following sleep loss than it was when subjects were not sleep deprived (Elkin \& Murray, 1974); and $d^{\prime}$ was very low if subjects saw the digit-triples in sequence on a computer screen but at the same time engaged in articulatory suppression (Murray, Rowan, \& Smith, 1988).

The set of experiments to be reported here was designed to demonstrate that a phenomenon true of immediate recall would also apply in immediate probed recognitionnamely, that lists of eight target digit-triples that were heard, voiced aloud by the subject as they were seen, or heard spoken by somebody else as they were seen would yield higher hit rates than would target items simply read silently as they were seen. Previous research on recall and on recognition had demonstrated the existence of what has come to be known as the modality effect or the auditory superiority effect. Murdock (1968) had reported that immediate probed recognition was superior if the presentation had an auditory component; and Greene (1988) had reported auditory superiority in a recognition task fol- 
lowing an auditory suffix. Auditory superiority effects in immediate recall following the voicing aloud of seen lists by the subjects themselves had been demonstrated by Murray (1966) and by Cantor and Engle (1989); when other people spoke the lists, auditory superiority effects in recall had been demonstrated by Murdock (1967) for probed recall, by Madigan (1971) and Routh (1976) for serial recall, by Murdock and Walker (1969) and Engle (1974) for free recall, and by Penney (1979) for recall following an auditory suffix. A review of many issues related to the modality effect was provided by Penney (1989), who suggested that auditory and visual processing could coexist as "separate streams" of processing in the subject's consciousness at any given moment.

However, only borderline auditory superiority effects were obtained in the probed recognition task used by Murray, Birch, Chase, Eyolfson, and Simms (1991). But in their experiments, the probe was always viewed silently, even though the eight target digit-triples had been mouthed, whispered, or voiced by subjects (Experiment 1 by Chase) or seen at the same time as the subjects heard the experimenter say them in a whispered, soft, or loud voice (Experiment 3 by Eyolfson).

One possible explanation for the lack of strong auditory superiority in that study would be based on the principle of encoding specificity (Tulving \& Thomson, 1973). It might be argued that, if the probe were to be processed (encoded) by the subject in exactly the same way as were the targets, the task would be generally easier, with a corresponding increase in the likelihood of obtaining an auditory superiority effect. Feenan and Snodgrass (1990) have demonstrated how keeping the context constant for both targets and probes can facilitate recognition, as compared with the case in which the context of the probes are incongruent with the context of the targets. However, it can sometimes happen that, in recognition tasks, encoding specificity fails to be demonstrated as reliably as it is in recall tasks for the same kind of material (Godden \& Baddeley, 1980; Smith, Glenberg, \& Bjork, 1978). Theories that attempt to account for these exceptions have been discussed by Baddeley (1990, chap. 11); it would appear that, if the context is inseparably associated with the target material, encoding specificity is more likely to be demonstrated in recognition tasks than if the context is weakly or separably associated with the target material.

In fact, encoding specificity effects with regard to modality (auditory vs. visual) have been demonstrated both in an immediate probed recognition task and in a task involving longer retention intervals. With respect to the former, Kirsner and Craik (1971) presented target lists of eight words, either visually or auditorially presented at a rate of one word per second, with the probe words also being either visually or auditorially presented at a delay of $2 \mathrm{sec}$ after the final target word. Words that were presented auditorially were associated with the highest hit rates, especially over serial positions 2-7; but whether the targets were visual or auditory, it was always the case that a probe presented in the same modality as the targets yielded a higher hit rate than that associated with the same serial position when the probe was in the different modality. Responses were also faster to auditory probes testing memory for auditory targets than for targets at corresponding serial positions in the other three conditions. However, FAs were kept at a minimum (fewer than $1 \%$ commission errors) in this study, and we shall see below that, in our study using digit-triples as the target material, the FA rates will be studied in the same detail as will hit rates.

With respect to recognition at longer retention intervals, Kirsner (1974) found that, in a continuous recognition task with retention intervals between repeated words varying from 1 to $32 \mathrm{sec}$, at all retention intervals the mean percentage of words recognized was higher when the two words were presented in the same modality than when they were presented in differing modalities. FA rates were higher than they were in the study of Kirsner and Craik (1971) but were still low by comparison with the FA rates to be reported below with digit-triples as the target material. In Experiment 1 of Kirsner, in which only old or new judgments were required as the list of words progressed continuously, the obtained FA rate was .105; in Experiment 2 , in which subjects judged not only whether each word was old or new but also whether, if it was judged to be old, it had appeared in the same modality as that associated with the first presentation of that word, the FA rate was .066 .

Another possible explanation for the failure of Murray et al. (1991) to obtain a significant modality effect would be based on the fact that recognition tasks are usually associated with a higher level of performance than is the case for recall tasks. Perhaps auditory superiority in recognition is harder to establish statistically than is the case for recall because of ceiling effects, leading to the conclusion that, if auditory superiority is to be convincingly demonstrated in immediate recognition, a rather large sample of data is required. Hence, the original purpose in starting the experiments of the present paper was to try to show that auditory superiority could be demonstrated in immediate probed recognition, not only when encoding specificity effects were controlled, but also when a larger number of subjects were tested than the 12 that had been tested by Murray et al. (1991). Logie, Della Sala, Laiacona, Chalmers, and Wynn (1996) have also reported that some well-established phenomena in the literature on immediate recall (e.g., the word length effect and the acoustic confusability effect) are not obtained by all subjects and that an important determinant of whether these effects are obtained is the strategy of subvocal rehearsal, which may vary from subject to subject. The issue of the reliability of data in experiments on immediate retrieval clearly also has to be raised in the context of data concerning immediate probed recognition performance.

Accordingly, we designed and carried out three experiments to examine whether the auditory superiority effect could be obtained in an immediate probed recognition task, using samples of subjects twice as large as those used 
by Murray et al. (1991) and attempting to control for effects related to the congruency of the sensory modality used for the targets with that of the probe. Three types of auditory conditions were compared: Lists of eight targettriples were heard (with no visual components), seen and simultaneously voiced aloud by the subject, or seen and, at the same time, heard by the subject as spoken by the experimenter's voice.

\section{METHOD}

In each of the three experiments, to be labeled hearing, seeingplus-voicing, and seeing-plus-hearing, the apparatus and procedure were identical, except for the manner in which the subjects perceived or encoded the target lists and their associated probes. Hence, we shall begin by describing the method of the hearing experiment and then indicate, where appropriate, how the seeing-plusvoicing and seeing-plus-hearing experiments differed in method from the hearing experiment.

\section{The Hearing Experiment}

Apparatus and Design. The target lists and probes consisted of digit-triples presented in orange one at a time on the black screen of a Zenith ZF-158-52 personal computer just to the right of a fixation point in the center of the screen. Each target list consisted of eight digit-triples randomly selected by the computer so that there was no replication of triples within the list. Each triple was on for $1 \mathrm{sec}$, followed by a 1-sec blank interval; thus, the presentation rate was one triple every $2 \mathrm{sec}$, a rather slow rate easily allowing for encoding into inner speech. The last item in each target list was followed by a beep accompanied by the visually presented word WAIT for half a second; then a probe triple appeared for as long as the subject took to give his recognition response. The probability that the probe was old was .5. The computer was preprogrammed so that the target in each serial position was probed equally often within an experimental session whose duration (in terms of the number of trials--i.e., lists presented) could be typed into the computer by the experimenter before each session.

Every session was devoted to one particular encoding condition. Following 16 practice trials, 96 trials were carried out by the subject, with 48 trials involving new probes mixed randomly with 48 involving old probes. With eight serial positions in each list, this meant that each subject was tested six times per serial position per encoding condition (session) with old probes. There were 24 subjects tested on each condition (see below). This meant that the hit rate reported in the Results section below for each of the eight serial positions for each encoding condition was based on $6 \times 24$ (i.e., 144) trials with old probes. The corresponding FA rate was based on $144 \times 8$ (i.e., 1,152) trials with new probes.

The design of the hearing experiment required each subject to come to the laboratory for four experimental sessions, each devoted to 96 trials under one of the four encoding conditions. The order in which each subject took the four conditions (across the four sessions) was determined by a counterbalanced design, so that each condition occurred equally often in the first, second, third, or fourth session and was followed equally often in the next session by any other of the four conditions. These conditions were as follows:

see/see: each target list was silently seen, followed by a silently seen probe;

see/hear: each target list was silently seen, followed by a probe heard as spoken by the experimenter (the computer screen being covered up in such a way that the subject could not see it);

hear/see: each target list was heard by the subject as spoken by the experimenter; then the subject saw the probe on the computer screen; hear/hear: the subject heard both the target list and the probe as spoken by the experimenter.

On condition see/hear, the subjects, after having seen the target list, moved their eyes very slightly to a position marked by an $\mathrm{X}$ on the wall just behind and immediately above the computer screen, while the experimenter moved a cardboard flap across the screen so that only she, and not the subject, could see the probe; on condition hear/see, the subjects heard the list while looking at the $\mathrm{X}$, then moved their eyes very slightly downward to see the probe on the now uncovered screen. On all conditions in which the experimenter read the digit-triples out loud, the triples were pronounced as separate digits - for example, 385 was pronounced "three, eight, five." We tried to make the transition from target to probe conditions as smooth and as undistracting as possible; this matter will be discussed later, in the context of Figure 3, below.

\section{Procedure}

On arrival, each subject was given written instructions concerning the old/new recognition task and then participated in the 16 practice trials using the encoding condition appropriate to that experimental session. They were instructed that, after they had perceived the eight target digit-triples, the beep and the word WAIT would indicate that the last digit-triple had been presented. When the probe-triple appeared, they should press a key on the computer keyboard to indicate whether they thought the probe was old or new. For half the subjects the old key was at the left of the keyboard, and for half it was at the right (which key was used by which subject was determined by prearranging that the old key was at the left for half the subjects whose first session was devoted to a particular condition and on the right for the other half, this being the case for each of the four conditions). A failsafe instruction was built into the design of the computer program so that, if a subject accidentally pressed a wrong key, they were informed of this on the screen and could make their response again. The subjects then indicated their confidence in rating their response by pressing a key on the number pad of their keyboard, with 1 indicating a complete guess and 7 indicating complete certainty. No particular instructions were given with respect to the speed of responding; the subject could report either old or new as quickly or slowly as he or she wished. In general, confidence ratings increased and reaction times decreased with the recency of the probed items. However, the reaction time data are not reported because, in any condition involving the subject's hearing the experimenter's saying the probe aloud, the subject's reaction time would be confounded with that of the experimenter. Although the confidence rating data are available for possible future analysis, they are not reported here, partly for reasons of space, partly because we shall not discuss signal detection theory in detail in this paper, and partly because Yonelinas $(1994,1997)$ has indicated that confidence ratings are determined in a manner more complex than was assumed by earlier writers such as Wickelgren and Norman (1966) and McNicol (1972), who derived their estimates of $d^{\prime}$ using confidence ratings.

At the end of each experimental session, a printout was obtained, showing many of the measures of central tendency that would be used in subsequent statistical analyses. These included mean hit rate for each serial position, mean FA rates, the number of times each confidence rating was used when old probes tested memory for a particular serial position or when new probes were involved, and others. We transformed hit rates and their corresponding FA rates into the signal detection measure $d^{\prime}$ by hand afterwards, using the tables of Elliott (1964); to obtain the mean $d^{\prime}$ value associated with any serial position under any encoding condition by any subject, the mean hit rate for that subject for those items probed at that serial position on that condition was entered into the table along with the mean FA rate for all lists (with new probes) for that subject and condition. Other measures of discrimination and bias were 
also examined, as will be made clear below at the appropriate points in the Results and Discussion sections.

On each trial, the probe remained on the screen until the subject had given his or her confidence rating, then disappeared; the next trial was signaled by the word REST appearing on the screen for $2 \mathrm{sec}$, followed by a $1-\mathrm{sec}$ pause. Then the the word READY appeared for $1 \mathrm{sec}$, followed by the first digit-triple of the next list. The subjects were instructed that, if they felt like a longer rest, they could delay giving their confidence rating on any trial. The typical duration of an experimental session was about $45 \mathrm{~min}$.

\section{Subjects}

The subjects were 24 volunteer undergraduates ( 13 females and 11 males) recruited by the experimenters; each subject came for four sessions spaced apart by at least a day. The experimenters were Andrea Leask and Melissa Wheeler, each of whom tested 12 subjects; it was necessary in practice to have different experimenters, because each used her data as the basis for an honors thesis and it was too much to ask one experimenter to test more than 12 subjects (for about $4 \mathrm{~h}$ each) for reasons of time constraints. It may be noted that, basing our conclusions on the results of power tests in which effect sizes were estimated on the basis of mean hit rates derived from Leask's 12 subjects only, we concluded that differences between mean hit rates (or $d^{\prime}$ values) obtained with different encoding conditions from 12 subjects only would be of borderline significance. This may have been a determinant of the borderline effects reported by Murray et al. (1991). The power tests were conducted in the manner suggested by Kraemer and Thiemann (1987, pp. 38-5I), making use of Leask's data, and the decision was therefore taken to double the number of subjects from 12 to 24 , the second 12 being tested by Wheeler.

\section{The Seeing-Plus-Voicing Experiment}

Apparatus and Design. The only difference between this experiment and the hearing experiment, apart from experimenters and subjects, was in the nature of the four encoding conditions. The encoding conditions were now:
see/see: as for the hearing experiment;
see/(see and voice): the subject silently saw the target list, but when the probe appeared, he or she spoke it aloud in a normal voice;
(see and voice)/see: the target list was spoken aloud by the subject; as each triple appeared it was pronounced as three digits (e.g., 249 would be voiced as "two-four nine"); however, when the probe appeared, it was silently seen but not voiced;
(see and voice)/ both the target list and the probe were seen and
(see and voice): $\quad$ voiced aloud by the subject.

Here, any distraction between target presentation and probe presentation will occur on the see/(see and voice) condition, in which the subject must be ready to voice the probe only, and the (see and voice)/see condition, in which the subject must be ready to stop voicing when the target list ends. The beep occupying the half-second interval between the target list and probe was also accompanied by the word WAIT on the screen, so there was no ambiguity about when the target list actually ended (the same held for all the experiments described here). Otherwise, the procedure was the same as that described for the hearing experiment.

Subjects. The seeing-plus-voicing experiment also involved 24 subjects, all volunteer undergraduates from Queen's University recruited by experimenters Leslie Dobell and Tricia Tate. The subjects were 17 females and 7 males.

\section{The Seeing-Plus-Hearing Experiment}

Apparatus and Design. These were exactly as those for the previous experiments, except that the four encoding conditions were now:

\begin{abstract}
see/see: $\quad$ as for the previous experiments
see/(see and hear): the target list was silently seen; when the probe appeared, the subject saw it but also heard the experimenter say it aloud; the experimenter was seated just behind and to the right of the subject and spoke the probe digit by reading it from the screen;

(see and hear)/see: the target list was seen at the same time as the experimenter spoke each digit, but the experimenter did not say anything when the probe appeared;

(see and hear)/ both the target list and the probe were seen by the (see and hear): subject and simultaneously heard as spoken by the experimenter.
\end{abstract}

In this experiment, any delay between the end of the target list and the presentation of the probe was minimal, because the subject did not need to do anything except continue to regard the screen; any distraction would be due to the sound of the experimenter's voice occurring only when the probe appeared [condition see/(see and hear)] or not occurring when the probe appeared [condition (see and hear)/see)].

Procedure. The procedure was the same as that for the hearing experiment.

Subjects. The seeing-plus-hearing experiment also involved 24 subjects, all volunteer undergraduates from Queen's University recruited by the experimenters Kimberley Burggraf and Nicole Boudreau. The subjects were 15 female and 9 males.

\section{RESULTS}

The auditory superiority effect will be demonstrated by a pattern shown by two particular curves on a graph in which serial position (or an equivalent, such as $i$, the number of items intervening between target and probe) is plotted on the abscissa and a measure of recognition performance on the ordinate (such measures could include hit rates, $d^{\prime}$ values, $d_{\mathrm{L}}$ values, or $P_{r}$ values; $d_{\mathrm{L}}$ is the analogue to $d^{\prime}$ associated with logistic, as opposed to normal, distributions of familiarity states, and $P_{r}$ values are measures of discrimination in the two-high-threshold model; both are described in detail by Snodgrass and Corwin, 1988). If such a serial position curve obtained for an encoding condition involving an auditory input yielded a significantly greater recency effect than did a corresponding serial position curve for a condition not involving an auditory component, this would be prima facie evidence for an auditory superiority effect.

Figure 1A shows such curves for mean hit rates for the four encoding conditions of the hearing experiment, Figure $1 \mathrm{~B}$ shows parallel data for the seeing-plus-voicing experiment, and Figure 1C for the seeing-plus-hearing experiment. It was deliberately decided to follow a convention established in the pioneering paper on immediate probed recognition for digit-triples (Wickelgren \& Norman, 1966)-namely, to label the abscissa on each graph of Figure 1 as representing the number of target items intervening between a particular item and the probe. This number, $i$, is normally zero for the final item of a list and increases to, for example, seven for the initial item of a list of eight. Thus, a hit rate that is highest at the left side of a curve relating mean hit rates to $i$ reflects a recency effect. The reason for making this decision was that, both in Wickelgren and Norman's model and in a partial match- 
ing theory of immediate probed recognition presented in a separate paper (Murray et al., 1998), the variable $i$ figures predominantly and the ordinal number representing serial position is relatively unimportant. In Figure 1, all curves involving auditory targets are shown as solid lines, and all those involving silently read targets are shown in dotted lines. It can easily be seen that, in all three experiments, there was a tendency for the solid lines to lie above the dotted lines on the left-hand side of the graph, which is evidence for the existence of auditory superiority effects in all three experiments. FA rates for each condition are shown at the right of each graph in Figure 1; the solid lines, representing conditions with auditory targets, are generally shorter than the dotted lines, indicating that auditory targets were associated with lower FA rates than were silently read targets.

Figure 2 shows the hit rates of Figure 1, converted into $d^{\prime}$ values by taking the hit rate shown for each subject for each serial position and the FA rate shown for that subject for that combination of target and probe modality (FA rate does not vary with serial position, as FA rates indicate the frequency of old responses given new probes) and looking up each combination of hit and FA rate so obtained in Elliott's (1964) tables of $d^{\prime}$. The means of these $d^{\prime}$ values were obtained by averaging across the 24 subjects in each condition. They were then plotted as in Figure 2 . It should be noted that this procedure is open to the objection that each curve of Figure 2 is simply a transformation of the corresponding curve of Figure 1, given a common FA rate. Nevertheless, it is clear that auditory superiority effects are as obvious to the eye in Figure 2, as they are in Figure 1.

For the hit rate data of Figure 1, there was a main effect of target modality on hit rates for $i=0$ to $i=4$ (the recency portion of the curve) that was statistically significant in each experiment [for the hearing experiment, $F(1,22)=9.35, M S_{\mathrm{e}}=0.0375, p<.01$; for the seeingplus-voicing experiment, $F(1,22)=20.58, M S_{\mathrm{e}}=0.0202$, $p<.01$; for the seeing-plus-hearing experiment, $F(1,22)=$ $\left.6.61, M S_{\mathrm{e}}=0.0775, p<.05\right]$. For the $d^{\prime}$ data of Figure 2, the corresponding $F$ values were even higher [for the hearing experiment, $F(1,22)=45.27, M S_{\mathrm{e}}=0.7176, p<$ .01 ; for the seeing-plus-voicing experiment, $F(1,22)=$ $34.2, M S_{\mathrm{e}}=0.8964, p<.01$; for the seeing-plus-hearing experiment, $\left.F(1,22)=14.75, M S_{\mathrm{e}}=1.8569, p<.01\right]$. If the analyses extended from $i=0$ to $i=7$-that is, all the serial positions were analyzed - the $F$ value for the seeing-plus-voicing data of Figure 1 remained significant $\left[F(1,22)=17.95, M S_{\mathrm{e}}=0.0318, p<.01\right]$, but the hit rates for the hearing and seeing-plus-hearing conditions yielded $F$ values that were not significant at the .05 level. However, the same analyses carried out on Figure 2 showed that there was an auditory superiority effect (demonstrated by a main effect of target modality) in the $d^{\prime}$ values that was significant in all three experiments. The hearing experiment yielded $F(1,22)=19.14, M S_{\mathrm{e}}=$ $1.105, p<.01$; the seeing-plus-voicing experiment yielded $F(1,22)=25.16, M S_{\mathrm{e}}=1.899, p<.01$; and the seeing-plus-hearing experiment yielded $F(1,22)=6.61$, $\left.M S_{\mathrm{e}}=2.071, p<.05\right]$.

A similar pattern was shown when other measures of discrimination were used. When the obtained hit and FA rates were used to calculate $d_{\mathrm{L}}$ and $P_{r}$ according to the formulae given by Snodgrass and Corwin (1988, p. 50), the main effect of target modality remained significant for both measures for $i=0$ to $i=4$ in all three experimentsthat is, the auditory superiority effect was clearly found for $d_{\mathrm{L}}$ and $P_{r}$ measures as well. For $i=0$ to $i=7$, there was a significant modality effect for $d_{\mathrm{L}}$ on all but the hearingand-seeing experiment and for $P_{r}$ on all three experiments.

The $d^{\prime}$ values of Figure 2 make use, of course, of the obtained FA values. Figure 3 shows the obtained FA values plotted in such a way as to show any crossover effects indicative of the operation of encoding specificity (or of a delay in encoding the probe). It is plain, not only that FA rates were considerably higher when the targets were merely seen than when the targets had an auditory component, but also that there was no consistent evidence for a crossover in any of the experiments. For that matter, there was no consistent evidence of a crossover for $i=0$ to $i=4$ in the hit rates either, as can be gathered from Figure 1, except for the hearing experiment, where the interaction between target modality and probe modality yielded $F(1,22)=20.08, M S_{\mathrm{e}}=0.0274, p<.01$. But this interaction failed to remain significant when the hit rate data were replotted as $d^{\prime}$ values. In general, this evidence, taken as a whole, suggests that encoding specificity played a minor role in determining both FA rates and hit rates in the present experiments. Its most striking appearance, in fact, turned out to be when $i=5$ to 7 in the seeing-plusvoicing experiment for hit rates $\left[F(1,22)=7.18, M S_{\mathrm{e}}=\right.$ $0.0347, p<.01]$; the corresponding $F$ ratios for $d^{\prime}$ values, $d_{\mathrm{L}}$ values, and $P_{r}$ values were also significant at the .01 level. That is, in the seeing-plus-voicing experiment only, there was good evidence for an encoding specificity effect on the primacy portion of the list only.

On the other hand, the main effect on FA rates of target modality was significant in the hearing experiment $\left[F(1,22)=7.51, M S_{\mathrm{e}}=0.007, p<.01\right]$ and in the seeingplus-voicing experiment $\left[F(1,22)=5.02, M S_{\mathrm{e}}=0.01\right.$, $p<.05]$. This main effect was not significant at the .05 level, however, in the seeing-plus-hearing experiment $\left[F(1,22)=2.63, M S_{\mathrm{e}}=0.011, p=.12\right]$.

Given that the presence of auditory cues during presentation reduces FA rates to this extent, it is not surprising that the evidence for a modality effect should be more compelling when $d^{\prime}$ values are used than when hit values are used. But what about response bias? Can it be claimed that the modality effect is in any way an artifact of a response bias, as opposed to being a genuine effect on memory storage? If the obtained hit and FA rates were used to calculate the bias measures $C_{\mathrm{L}}$ (the signal detection measure of bias assuming logistic distributions of familiarity states) and $B_{r}$ (the two-high-threshold measure of bias), analyses of variance (ANOVAs) showed that in none of the three experiments was there a main effect of target modal- 
A

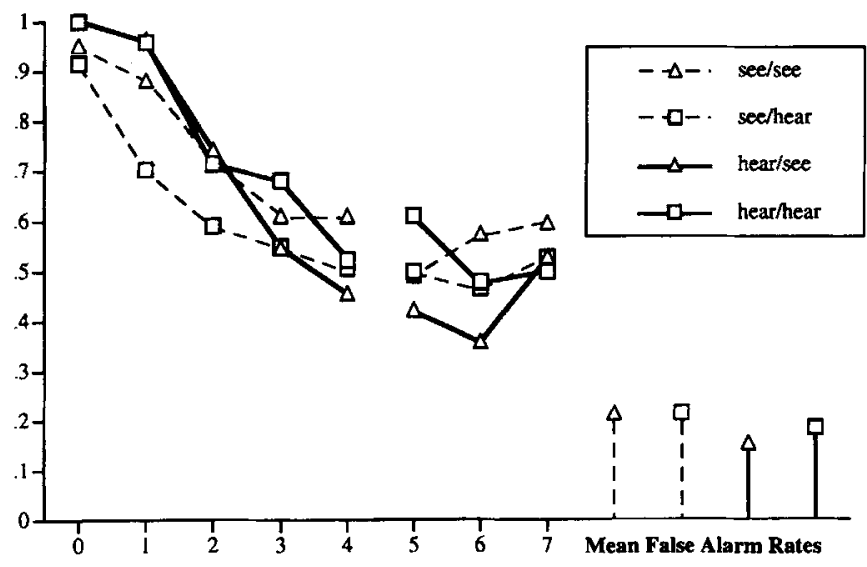

$\mathbf{B}$

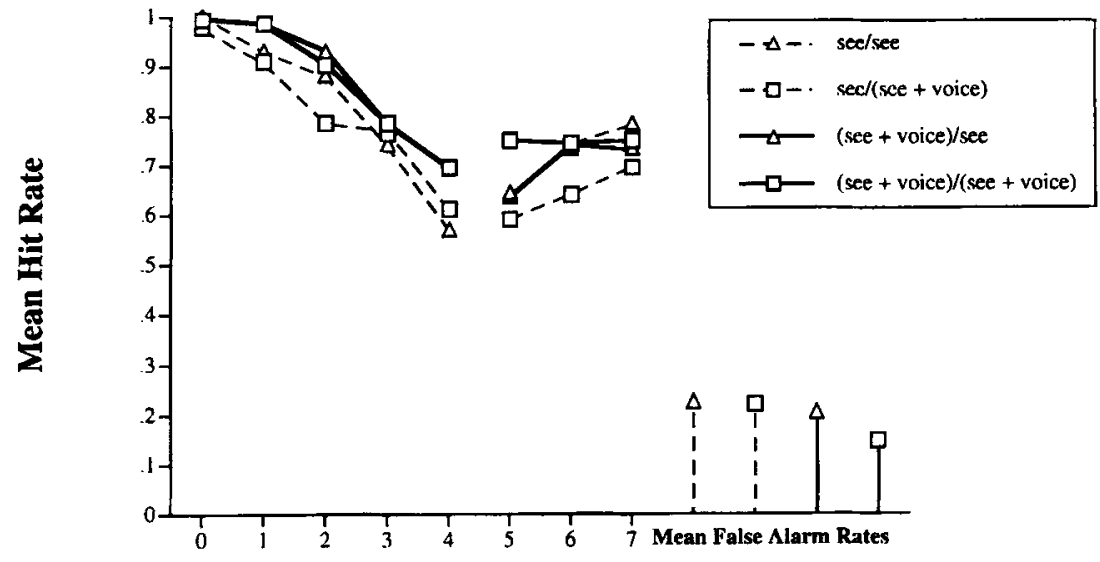

C

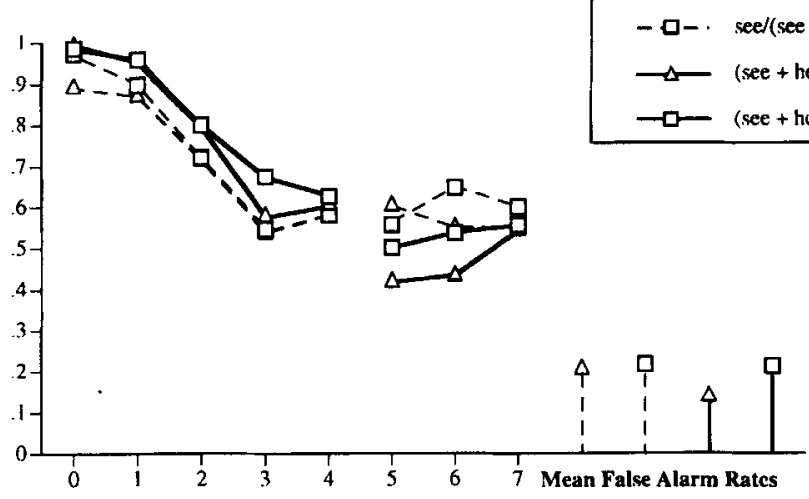

$i$ (number of items between target and probe)

Figure 1. Mean hit rates as a function of $i$, the number of target items intervening between a target and the probe, for the hearing experiment, shown in graph $A$, the seeing-plus-voicing experiment, shown in graph $B$, and the seeing-plus-hearing experiment, shown in graph $C$. Mean false-alarm rates are also shown.

ity on either of these measures for $i=0$ to 4 or for $i=0$ to 7 .

There were, of course, some other main effects and interactions of significance in the 57 ANOVAs that were actually carried out on the present data ( 3 experiments $X$
3 ranges of serial position $\times 6$ measures of performance, plus the 3 ANOVAs on FA rates, 1 for each experiment), but they appear to be of minor importance in terms of the theoretical arguments of this paper. More details of these ANOVAs are available on request from the first author. 
$\mathbf{A}$
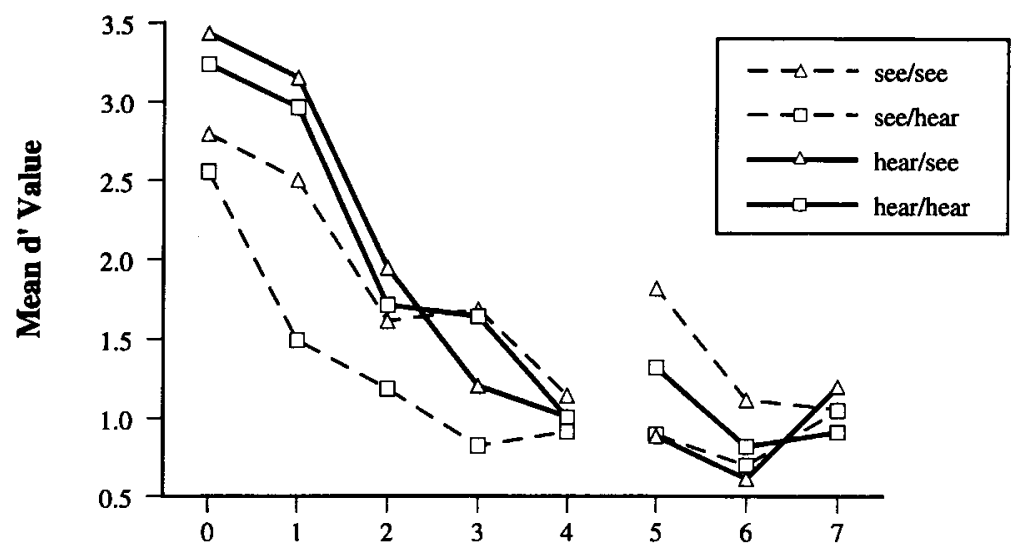

B

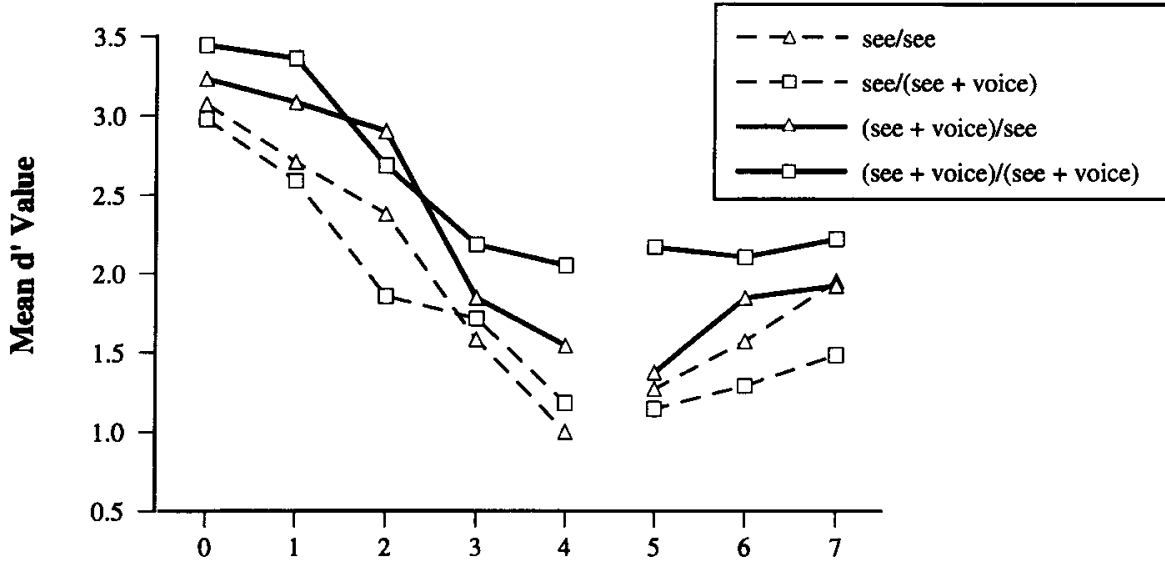

$\mathbf{C}$

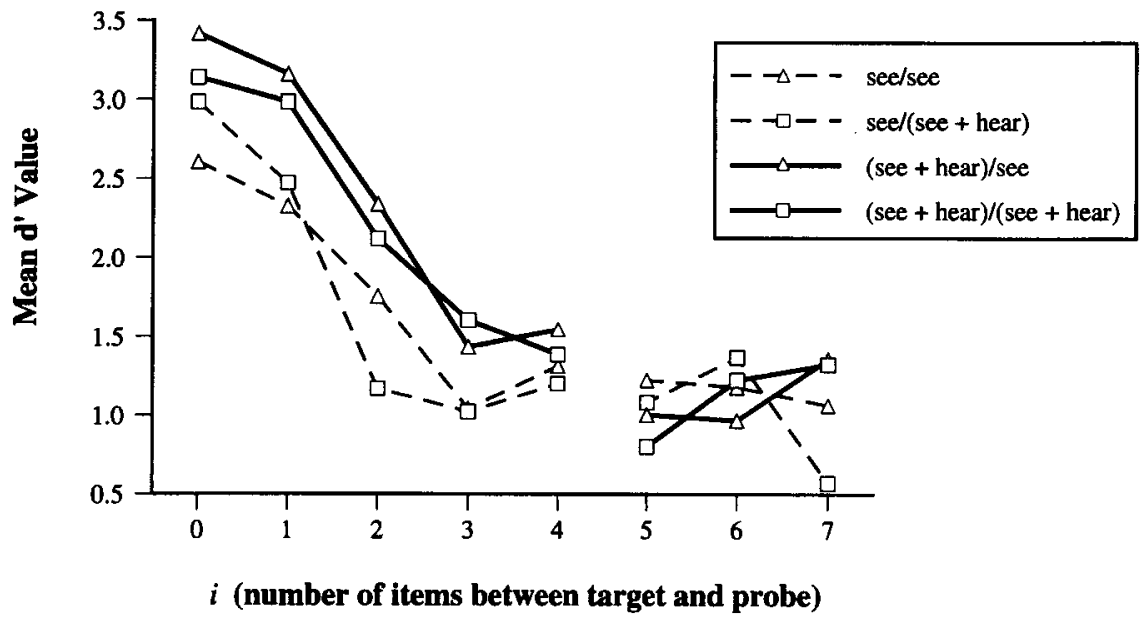

Figure 2. Mean $d^{\prime}$ values plotted in the format used for Figure 1.

\section{DISCUSSION}

Before discussing possible explanations for the above patterns of data, we wished to establish whether seen targets had yielded higher FA rates than had heard targets in earlier studies from our laboratory that had used au- ditory targets. We therefore reevaluated the data from some earlier experiments on probed recognition.

There were four such experiments involving heard targets, those of R. Chase, B. Eyolfson, L. Stanford, and S. Guger. The experiments of Chase and Eyolfson were reported as Experiments 1 and 3 of Murray et al. (1991); 
(a)

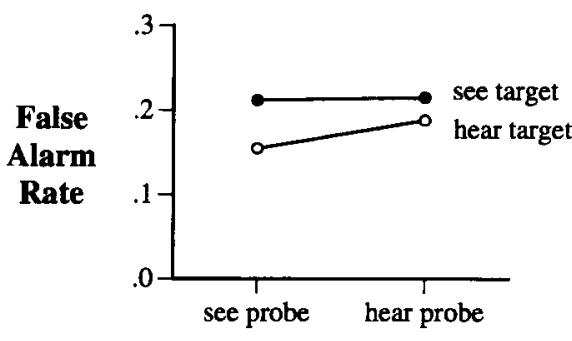

Hearing experiment (b)

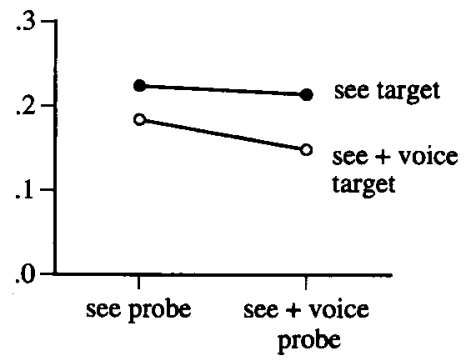

Seeing-plus-Voicing experiment (c)

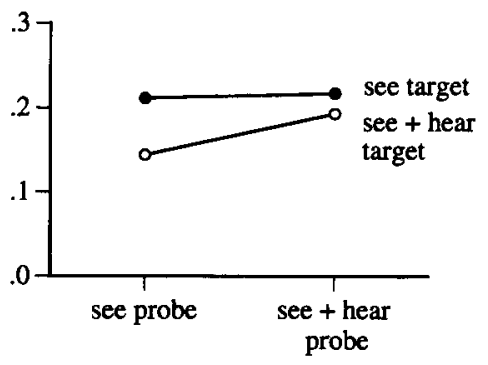

Seeing-plus-Hearing experiment

Figure 3. The effects of target modality and probe modality on false-alarm rates in the hearing, seeing-plus-voicing, and seeing-plushearing experiments.

using the same material (eight digit-triples per list) and computer method of presentation as those described above, Chase had each of 12 subjects silently read, silently mouth, whisper aloud, or voice aloud the target lists; on all the trials, the probe was silently seen. Stanford's experiment, here reported for the first time, was an exact replication of Chase's experiment, but her 12 subjects encoded the probe in the same way that they had encoded the immediately preceding target list. In Eyolfson's experiment, his 12 subjects silently read the targets or saw them while the experimenter whispered them or spoke them in a soft voice or a loud voice; again, all the probes were silently read. In Guger's study, here reported for the first time, her 12 subjects replicated Eyolfson's conditions but with the probe's being seen and/or heard in the same way as the corresponding target list. The experiments of Stanford and Guger represented attempts at exploring encoding specificity in probed recognition and were carried out before the three experiments of the present paper; the latter three experiments had subsequently been designed deliberately so that interactions between target modality and probe modality could be studied with an orthogonal design.

Table 1 summarizes the obtained hit rates (both for $i=0$ to 4 and for $i=0$ to 7) and FA rates for the experiments of Chase, Eyolfson, Stanford, and Guger, each of whom used 12 subjects, and for the hearing, seeing-plus-voicing, and seeing-plus-hearing experiments, each of which used 24 subjects. The mean hit and FA rates are subdivided, within each experiment, into means for seen targets as contrasted with means for heard targets. Next to each such pair is indicated whether the difference between those two means was statistically significant. By looking at the column headed "Mean FA Rate," the reader can see that FA rates were higher for seen than for heard targets in six of the seven experiments, significantly so in four of the former. It can also be seen from the final two columns that there was a mirror effect in 9 of the 14 cells of these columns. By mirror effect, we mean that, if the heard targets yielded a higher hit rate than did the seen targets, they also yielded a lower FA rate than did the seen targets. This pattern of hit and FA rates is often found in recogni- tion experiments, as was stressed by Glanzer and Adams (1985). However, it was only for the hearing and seeingplus-voicing experiments in the present paper-and not for any of the experiments conducted by Chase, Eyolfson, Stanford, or Guger - that the mirror effect was unambiguous. By this, we mean that the original hit rates obtained for seen as opposed to heard targets themselves differed significantly, as did the FA rates. It is important to state whether any mirror effect is based on data in which the two conditions concerned have significantly different hit and FA rates, in order to exclude mirror effects that come about by chance when two conditions have insignificantly different hit and FA rates.

If one were to make the assumption that the probability of a mirror effect in any one cell was .5 , the probability of obtaining nine mirror effects in the 14 cells of the final two columns of Table 1 would indeed be within the range of chance operation-namely, .212. But it appears to inspection that the probability of obtaining a mirror effect in any 1 cell is not .5 but depends on the number of subjects in the experiment of interest. Table 1 demonstrates very clearly that it was essential to have carried out the three experiments of this paper with 24 subjects in order to establish, not merely that heard targets were associated with higher hit rates than were seen targets in our study of immediate probed recognition (the modality effect), but also that heard targets were associated with lower FA rates than were seen targets in that study (giving rise to a mirror effect).

According to the theory of Glanzer and Adams (1985) and Glanzer, Adams, Iverson, and Kim (1993), most (perhaps all) mirror effects obtained in recognition experiments can be accounted for in terms of a modification of signal detection theory named attention/likelihood theory. The recognition task mainly studied by these authors was the mixed test list paradigm, involving supraspan lists of words varying in frequency. However, we believe that the above demonstration that, in the immediate probed recognition paradigm, heard targets generally yield lower FA rates than do seen targets and, therefore, that a comparison of the two conditions yields a 
Table 1

An Indication of Whether Mirror Effects Were Revealed When Hit and False-Alarm (FA) Rates for Seen as Opposed to Heard Targets Were Compared in Seven Experiments

\begin{tabular}{|c|c|c|c|c|c|c|c|}
\hline Experiment & Number of Subjects & Targets & $\begin{array}{c}\text { Mean Hit Rate } \\
(i=0 \text { to } 4)\end{array}$ & $\begin{array}{c}\text { Mean Hit Rate } \\
(i=0 \text { to } 7)\end{array}$ & Mean FA Rate & $\begin{array}{c}\text { Mirror Effect? } \\
(i=0 \text { to } 4)\end{array}$ & $\begin{array}{c}\text { Mirror Effect? } \\
(i=0 \text { to } 7)\end{array}$ \\
\hline \multirow[t]{2}{*}{ Chase } & 12 & Seen & .786 & .678 & $.2555^{*}$ & No & No \\
\hline & & Heard & .7705 & .6745 & .1875 & & \\
\hline \multirow[t]{2}{*}{ Eyolfson } & 12 & Seen & .734 & .689 & $.270 \dagger$ & Yes & No \\
\hline & & Heard & .772 & .658 & .1873 & & \\
\hline \multirow[t]{2}{*}{ Stanford } & 12 & Seen & $.7835^{*}$ & .688 & .1775 & No & No \\
\hline & & Heard & .8125 & .7005 & .1815 & & \\
\hline \multirow[t]{2}{*}{ Guger } & 12 & Seen & .764 & .675 & .260 & Yes & Yes \\
\hline & & Heard & .813 & .695 & .1736 & & \\
\hline \multirow[t]{2}{*}{ Hearing } & 24 & Seen & $.7045+$ & .643 & $.2135 t$ & Yes & Yes \\
\hline & & Heard & .759 & .6595 & .1715 & & \\
\hline \multirow[t]{2}{*}{ Seeing-plus-voicing } & 24 & Seen & $.817 \dagger$ & $.743 \dagger$ & $.219^{*}$ & Yes $*$ & Yes $\neq$ \\
\hline & & Heard & .8735 & .8165 & .1665 & & \\
\hline \multirow[t]{2}{*}{ Seeing-plus-hearing } & 24 & Seen & $.733^{*}$ & .680 & .214 & Yes & Yes \\
\hline & & Heard & .7995 & .6925 & .1685 & & \\
\hline
\end{tabular}

mirror effect can also be explained in terms of grouping strategies. An explicit comparison of this grouping theory with the attention/likelihood theory of Glanzer and his co-workers will be found in the paper by Murray et al. (1998). Here, we shall restrict our remarks to claiming that the mirror effect associated with seen as opposed to heard targets can plausibly be accounted for just as well in terms of different grouping strategies and that the present data are consistent in this respect with some data previously reported by Murray et al. (1998).

The first step in the argument is to ascertain what it is about auditory inputs that makes them less prone to be associated with FAs than are seen inputs. A strong hint has been given by the research of Frankish (1985), who showed that auditory targets benefit particularly from temporal grouping, and of Macken and Jones (1995), who argued that auditory inputs in general, but particularly auditory inputs with associated articulatory movements, leave memory representations to which can be attributed the quality that the relevant contents of the targets are better segmented; moreover, Macken and Jones claimed that, within those segments, individual sequences of sounds are better grouped than is the case for visual presentation. Temporal grouping itself, whose advantages for immediate recall were stressed by Ryan (1969a, 1969b) and by Murray (1995, pp. 123-128), is associated with wider pauses between groups than within groups. This may ensure that each group is more distinctive from the background of the rest of the list; evidence supporting the notion that retrieval efficiency in immediate recall is indeed a function of the distinctiveness of each item in the list, as determined by the spacing of items from their neighbors, has been provided by Crowder and Neath (1991). Neath (1993) has also shown that delaying the probe by $10 \mathrm{sec}$ has the effect of reducing the role of recency and enhancing the role of primacy in the probed recognition of visual patterns (snowflakes); he showed that the obtained data can be predicted by a model that includes a reduction in the predominance of recency over primacy as the retention interval increases, a model in which the relative distinctiveness of individual memory items is a key parameter.

However, temporal grouping is not the only form of grouping; grouping by similarity between temporally nonadjacent items can also occur. Neath and Nairne (1995) explicitly assume that a later target can overwrite an earlier target that is similar to it. If articulatory suppression is present as the target material is heard, Neath and Nairne believe that the suppression task adds a string of extra sounds into the sample of possible responses that the subject can draw from in secondary memory, his or her goal being to choose from this sample the item most similar to an auditory trace in primary memory that has been degraded because of having been overwritten by one or more later, similar-sounding items. Nairne (1990) had earlier shown that the elimination of the acoustic confusability effect for lists seen with concurrent articulatory suppression could be well modeled by a computer simulation in which auditory elements played a much larger role in the internal representation than did visual elements.

These contributions by others lead us to believe that the low FA rate associated with auditory targets should be considered to be a by-product of the fact that auditory targets are in themselves well grouped, with the grouping being particularly enhanced if the targets are voiced by the subject. The evidence for this last assertion comes from the fact that auditory superiority effects were restricted in the hearing and in the seeing-plus-hearing experiments to the final parts of the target list but extended throughout the target list in the case of the seeing-plus-voicing experiment. Subsidiary evidence supporting the general idea that it is the memory representations provided by the subject that constitute the basis of matching in the recognition process for alphanumeric stimuli comes from the 
report by Surprenant and Neath (1996) that the recall of vowels and consonants (in various combinations) is based on the discriminability of their corresponding memory representations, rather than on the discriminability of their acoustic properties as measured by identification tasks.

If it is true that digit-triples that are passively heard or heard via voicing are well grouped, then, when an old probe appears, activation of the required trace will be immediate and involve the whole group-that is, the three digits of the triple. Moreover, if a new probe appears, it can probably be judged to be new because, even though, say, there may be an accidental match between the first digit of the new probe and the first digit of an (auditory) target's trace, the rest of that composite trace is so well grouped with the trace of the first digit that the remaining two digits of the new probe will not match the final two digits of that trace, and the probe will accordingly be judged correctly to be new.

But if the target list is silently seen, the lack of an auditory component will mean that each triple may not be encoded as a clear group; for example, the first digit of a silently seen triple may be well encoded in inner speech, but the other two digits less well encoded within the composite representing the triple. Hence, an old probe may lead to missed signals, because the match between the probe's composite trace and the corresponding loosely bound composite target's trace may be poor enough for the subject to mistakenly think that the probe is new, whereas a new probe, whose first digit accidentally matches the first digit of a loosely bound composite target trace of a triple, may be mistakenly judged to be old, with the subject hoping that there is also a match between the final two digits of the new probe and the (barely retained) final two digits of the target's trace. If articulatory suppression took place concurrently with the viewing of the targets, attempted encoding in memory of parts of digittriples (as opposed to whole digit-triples) would be even more likely, leading both to a reduction in hits, as compared with a condition involving auditory target traces with no articulatory suppression, and, at the same time, to an increase in FAs, owing to the subjects' responding old when there was an accidental match between part of a new probe (say, its first digit) and part of a poorly encoded trace of a target-triple (say, its first digit). But if this is the case, we have an explanation of the mirror effect that is obtained when conditions involving auditory targets are compared with conditions involving visual targets: The latter targets are associated with more partial encoding, hence lower hit rates and higher FA rates, than was the case with auditory targets.

As thus stated in words, this explanation of why auditory targets are associated both with higher hit rates than are silently viewed targets (the modality effect) and with lower FA rates (yielding a mirror effect for the task of immediate probed recognition) lacks rigor. However, two reasons may be given for asserting its value in the context of the present yes/no recognition tasks.
First, Murray et al. (1998) have argued that this theory can, in fact, be made rigorous, insofar as it can be encapsulated in some analytic equations involving probability. They also have argued that the theory has received support from some new experiments in which list length was varied from five to eight digit-triples, the preset probability of an old probe varied between $.33, .5$, and .67, and the role of audition was varied by having subjects hear, see-and-voice, or see-and-hear the target lists as they were presented or view them with concurrent auditory suppression. The equations predicting hit and FA rates incorporate a variable named $m$, which is the probability that the first digit of any triple matches the first digit of the previous triple. In their paper, and in the three experiments of the present paper using 24 subjects, $m$ was equal to .111 , or one ninth (the digits ranged from 1 to 9); in the earlier experiments of Chase, Eyolfson, Stanford, and Guger with 12 subjects, $m$ was equal to .10 (the digits ranged from 0 to 9). Murray et al. (1998) also argued that this distributionfree partial matching theory can be elaborated in such a way as to yield a rational response theory modified to include partial matching. This latter theory, it was claimed, can account for mirror effects obtained in the mixed test list paradigm that uses words of low or high frequency, the paradigm originally examined by Glanzer and Adams (1985). Partial matching theory was held to be applicable to lists, the number of whose partially encoded traces was below the memory span; modified rational response theory was more applicable to long lists that demanded guessing.

Second, the mean obtained FA rate (.1922) in the three experiments with 24 subjects reported here exceeds by far the FA rate of .01 obtained by Kirsner and Craik (1971) in their first experiment (using an immediate probed recognition task) and the mean FA rate of .0855 obtained by Kirsner (1974) in his two experiments (using a continuous recognition task). Kirsner and Craik also studied immediate probed recognition of target lists consisting of eight stimuli, but their stimuli were words rather than digittriples. If the theory of Murray et al. (1998) is correct, this difference in mean FA rates between Kirsner's studies and ours might have been predicted, because digit-triples constructed randomly do not form meaningful unified memory entities in the way that words do. Although indeed, it might be possible to demonstrate FAs from partial matching between new probes and stored memory representations of targets consisting of words, in the way that Baddeley (1966) could demonstrate recall errors when the target list consisted of six 3-letter words that were acoustically confusable, it is unlikely that the target and probe words used by Kirsner and Craik (1971) were confusable in this sense; their target words consisted of eight 5-, 6-, or 7-letter two-syllable nouns. Hence, the FA rates obtained in the experiments reported in the present paper were, we suggest, higher than those reported by Kirsner and Craik because we used digit-triples that were easily decomposable into partial memory representations, 
which was probably not the case with the two-syllable nouns used by those authors.

We conclude, therefore, by saying that, in this paper, we have asserted that, provided sufficient data are supplied for unwanted effects from random interlist and intersubject variability to be reduced to an acceptable level, the immediate probed recognition task can be shown to reveal, not only an auditory superiority (modality) effect, but also a mirror effect, insofar as the hit rates and FA rates associated with seen as opposed to heard targets are concerned. It has been argued that the higher FA rate associated with seen as opposed to heard targets can be accounted for in a psychological manner (namely, as a byproduct of a grouping strategy). This is not to say that other interpretations of this finding need be completely discounted; but in the partial matching theory described by Murray et al. (1998), it is argued that the psychological explanation of the lower FA rates associated with seen as opposed to heard targets can serve as a starting point for a distribution-free partial matching theory of yes/no recognition processes that has at least some initial plausibility as an alternative to more conventional theories based on assumptions about the distributions of the states of familiarity believed to underlie yes/no recognition judgments.

\section{REFERENCES}

BADDELEY, A. D. (1966). Short-term memory for word sequences as a function of acoustic, semantic and formal similarity. Quarterly Journal of Experimental Psychology, 18, 362-365.

Baddeley, A. D. (1990). Human memory: Theory and practice. Boston: Allyn \& Bacon.

CANTOR, J., \& ENGLE, R. W. (1989). The influence of concurrent load on mouthed and vocalized modality effects. Memory \& Cognition, 17, 701-711.

Crowder, R. G., \& Neath, I. (1991). The microscope metaphor in human memory. In W. E. Hockley \& S. Lewandowsky (Eds.), Relating theory and data: Essays on human memory in honor of Bennet $B$. Murdock (pp. 111-125). Hillsdale, NJ: Erlbaum.

ElKIN, A. J., \& MurRaY, D. J. (1974). The effects of sleep loss on shortterm recognition memory. Canadian Journal of Psychology, 28, 192 198.

ELLIOTT, P. B. (1964). Tables of $d^{\prime}$. In J. A. Swets (Ed.), Signal detection and recognition by human observers (pp. 651-684). New York: Wiley.

ENGLE, R. W. (1974). The modality effect: Is precategorical acoustic storage responsible? Journal of Experimental Psychology, 102, 824-829.

FeEnan, K., \& SNOdgrass, J. G. (1990). The effect of context on discrimination and bias in recognition memory for pictures and words. Memory \& Cognition, 18, 515-527.

Frankish, C. (1985). Modality-specific grouping effects in short-term memory. Journal of Memory \& Language, 24, 200-209.

Glanzer, M., \& ADAMS, J. K. (1985). The mirror effect in recognition memory. Memory \& Cognition, 13, 8-20.

Glanzer, M., Adams, J. K., Iverson, G. J., \& Kim, K. (1993). The regularities of recognition memory. Psychological Review, 100, 546-547.

GoDDEN, D., \& BADDELEY, A. D. (1980). When does context influence recognition memory? British Journal of Psychology, 71, 99-104.

GREENE, R. L. (1988). Stimulus suffix effects in recognition memory. Memory \& Cognition, 16, 206-209.

KIRSNER, K. (1974). Modality differences in recognition memory for words and their attributes. Journal of Experimental Psychology, 102, 579-584.
KIRSNER, K., \& CraIK, F. I. M. (1971). Naming and decision processes in short-term recognition memory. Journal of Experimental Psychology, 88, 149-157.

Kraemer, H. C., \& ThIemann, S. (1987). How many subjects? Statistical power analysis in research. Beverley Hills: Sage.

Logie, R. H., Della Sala, S., Laiacona, M., Chalmers, P., \& Wynn, V. (1996). Group aggregates and individual reliability: The case of verbal short-term memory. Memory \& Cognition, 24, 305-321.

MACKEN, W. J., \& JONES, D. M. (1995). Functional characteristics of the inner voice and the inner ear: Single or double agency? Journal of Experimental Psychology: Learning, Memory, \& Cognition, 21, 436-448.

MADIGAN, S. A. (1971). Modality and recall order interactions in shortterm memory for serial order. Journal of Experimental Psychology, 87, 294-296

MCNICOL, D. (1972). A primer of signal detection theory. London: George Allen \& Unwin.

MURDOCK, B. B., JR. (1967). Auditory and visual stores in short-term memory. Acta Psychologica, 27, 316-324.

MURDOCK, B. B., JR. (1968). Modality effects in short-term memory: Storage or retrieval? Journal of Experimental Psychology, 77, 79-86.

MURDOCK, B. B., JR., \& WALKER, K. D. (1969). Modality effects in free recall. Journal of Verbal Learning \& Verbal Behavior, 8, 665-676.

MuRRaY, D. J. (1966). Vocalization-at-presentation and immediate recall, with varying recall methods. Journal of Experimental Psychology, 18, 9-18.

MURRAY, D. J. (1995). Gestalt psychology and the cognitive revolution. London: Harvester Wheatsheaf.

Murray, D. J., Birch, D. E., Chase, R. S., Eyolfson, B. H., \& Simms, K. A. M. (1991). The effect of vocalization at presentation on shortterm recognition of three-digit numbers. Canadian Journal of Psychology, 45, 462-473.

Murray, D. J., Burhop, J., Centa, S., Chande, N., Oinonen, K., Thomas, T., Wilkie, T., \& Farahmand, B. (1998). A partial matching theory of the mirror effect in immediate probed recognition. Memory \& Cognition, 26, 1196-1213.

MurRaY, D. J., \& HiTCHCOCK, C. H. (1974). The nature of the memory deficit in Korsakoff's psychosis. Canadian Journal of Psychology, 27, 414-421

MurRaY, D. J., Rowan, A. J., \& Smith, K. H. (1988). The effect of articulatory suppression on short-term recognition. Canadian Journal of Psychology, 42, 424-436.

NAIRNE, J. S. (1990). A feature model of immediate memory. Memory \& Cognition, 18, 251-269.

NEATH, I. (1993). Distinctiveness and serial position effects in recognition. Memory \& Cognition, 21, 689-698.

NeATH, I., \& NaIRNE, J. S. (1995). Word-length effects in immediate memory: Overwriting trace decay theory. Psychonomic Bulletin \& Review, 2, 429-441

PENNEY, C. G. (1979). Interactions of suffix effects with suffix delay and recall modality in serial recall. Journal of Experimental Psychology: Human Learning \& Memory, 5, 507-521.

PenNey, C. G. (1989). Modality effects and the structure of short-term verbal memory. Memory \& Cognition, 17, 398-422.

Routh, D. A. (1976). An across-the-board modality effect in immediate serial recall. Quarterly Journal of Experimental Psychology, 28, 285-304

Ryan, J. (1969a). Grouping and short-term memory: Different means and patterns of grouping. Quarterly Journal of Experimental Psychology, 21, 137-147.

RYAN, J. (1969b). Temporal grouping, rehearsal and short-term memory. Quarterly Journal of Experimental Psychology, 21, 148-155.

Smith, S. M., Glenberg, A., \& BJork, R. A. (1978). Environmental context and human memory. Memory \& Cognition, 6, 342-353.

SNODGRass, J. G., \& CoRwIN, J. (1988). Pragmatics of measuring recognition memory: Applications to dementia and amnesia. Journal of Experimental Psychology: General, 117, 34-50.

Surprenant, A., \& NeATH, I. (1996). The relation between discriminability and memory for vowels, consonants, and silent-center vowels. Memory \& Cognition, 24, 356-366. 
Tulving, E., \& Thomson, D. M. (1973). Encoding specificity and retrieval processes in episodic memory. Psychological Review, 80, 352373.

Wickelgren, W. A., \& Norman, D. A. (1966). Strength models and serial position in short-term memory. Journal of Mathematical Psychology, 3, 316-347.

YoNELINAS, A. P. (1994). Receiver-operating characteristics in recognition memory: Evidence for a dual-process model. Journal of Ex- perimental Psychology: Learning, Memory, \& Cognition, 6, 13411354.

YoNeLINAS, A. P. (1997). Recognition memory ROCs for item and associative information: The contribution of recollection and familiarity. Memory \& Cognition, 25, 747-763.

(Manuscript received April 2, 1997; revision accepted for publication March 12, 1998.) 